\title{
Momentum and Energy Transfer in an lonospheric Critical Ionization Velocity Experiment
}

\author{
Bolin, O.; Brenning, N.; Swenson, C. M.; Primdahl, Fritz
}

Published in:

Physical Review Letters

Link to article, DOI:

10.1103/PhysRevLett.75.3673

Publication date:

1995

Document Version

Publisher's PDF, also known as Version of record

Link back to DTU Orbit

Citation (APA):

Bolin, O., Brenning, N., Swenson, C. M., \& Primdahl, F. (1995). Momentum and Energy Transfer in an Ionospheric Critical lonization Velocity Experiment. Physical Review Letters, 75(20), 3673-3676.

https://doi.org/10.1103/PhysRevLett.75.3673

\section{General rights}

Copyright and moral rights for the publications made accessible in the public portal are retained by the authors and/or other copyright owners and it is a condition of accessing publications that users recognise and abide by the legal requirements associated with these rights.

- Users may download and print one copy of any publication from the public portal for the purpose of private study or research.

- You may not further distribute the material or use it for any profit-making activity or commercial gain

- You may freely distribute the URL identifying the publication in the public portal 


\title{
Momentum and Energy Transfer in an Ionospheric Critical Ionization Velocity Experiment
}

\author{
O. Bolin, ${ }^{1}$ N. Brenning, ${ }^{1}$ C. M. Swenson, ${ }^{2}$ and F. Primdahl ${ }^{3}$ \\ 'Alfvén Laboratory, Royal Institute of Technology, Stockholm, Sweden \\ ${ }^{2}$ Department of Electrical Engineering, Utah State University, Logan, Utah 84322 \\ ${ }^{3}$ Danish Space Research Institute, Lyngby, Denmark
}

(Received 24 March 1995)

\begin{abstract}
We present new data from the subpayload of the CRIT II ionospheric active injection experiment. The analysis made possible by these data provides a good understanding of the momentum transfer between the injected ions and the ambient ionosphere. It resolves the conflict between the two competing models for the energy transfer from the newly created ions to hot electrons, while also giving a natural coupling between the energy and momentum transfer processes.
\end{abstract}

PACS numbers: 52.25.Jm, 52.25.Ya, 94.20.Vv

The CRIT II rocket was launched in 1989 to study the critical ionization velocity (CIV) phenomenon originally proposed by Alfvén [1]. At an altitude of approximately $400 \mathrm{~km}$, two shaped charge explosions created beams of neutral barium. The interaction between these barium beams and the ambient ionosphere was studied by two separate payloads. Both payloads were equipped with instruments for full three dimensional measurements of the electric and magnetic fields, as well as with charged particle detectors. The neutral beams were aimed directly at the main payload, while the subpayload was located outside of the beams, close to the same magnetic field line as the main payload. Some key parameters of the experiment are listed in Table I.

The experiment performed very well, and is unique in the sense that it for the first time ever produced data from two points on the same magnetic field line in an active injection experiment in the ionosphere.

Various results from the CRIT II experiment, mainly from the main payload, have been discussed previously [2-5]. However, we present here for the first time results based on the processed electric and magnetic field data from the subpayload, combined with data from the main payload. The subpayload data have made possible a full analysis of the momentum transfer between the newly ionized barium and the ambient ionospheric plasma, and to some extent also the energy transfer from the barium ions to the electrons, a necessary condition for CIV to occur.

We will present some of the most interesting magnetic field data from the subpayload. These data confirm

TABLE I. Some key parameters in the CRIT II experiment.

\begin{tabular}{cc}
\hline \hline $\mathbf{B}_{\mathrm{amb}}$ & $0.43 \times 10^{-4} \mathrm{~T}$ \\
$n_{\mathrm{amb}}\left(\mathrm{O}^{+}\right)$ & $56 \times 10^{10} \mathrm{~m}^{-3}$ \\
$V_{A}$ & $310 \mathrm{~km} \mathrm{~s}^{-1}$ \\
$v_{\mathrm{Ba}^{+}}$ & $8-13.5 \mathrm{~km} \mathrm{~s}^{-1}$ \\
$r_{L, \mathrm{Ba}^{+}}$ & $190-380 \mathrm{~m}$ \\
$f_{g, \mathrm{Ba}^{+}}$ & $4.8 \mathrm{~Hz}$ \\
\hline \hline
\end{tabular}

$0031-9007 / 95 / 75(20) / 3673(4) \$ 06.00$ the description of the momentum transfer given in [2] and [6]. Based on this, we will then proceed to study the currents perpendicular to the ambient magnetic field and use the results to model the coupling between the energy and momentum transfer mechanisms, and to resolve the conflict between the two present models for the energy transfer: the homogeneous model [7] and the inhomogeneous, or ionizing front, model [8]. Common to both these models is that the ionization is caused by electrons that have been collectively heated through an instability-driven process. In the homogeneous model the instability has a wave vector $\mathbf{k}$ that is parallel to the relative motion between the neutral gas and the ambient plasma, while in the inhomogeneous model $\mathbf{k}$ is perpendicular to that relative motion. The analysis will be focusing mainly on the low-frequency part of the data.

The raw data from the CRIT II electric and magnetic field experiments have been extensively processed in order to remove, or at least minimize, effects due to, e.g., dc offsets in the instruments, and to the spinning and coning of the payloads. The processing methods that have been used are described in [5] and [9]. The full electric and magnetic field data set from CRIT II will be published in the near future.

The coordinate system we will use in this paper is the magnetic coordinate system, as defined in [5]. In that system the $z$ axis is antiparallel to the ambient magnetic field, the $y$ axis is parallel to $\mathbf{v}_{\text {beam }} \times \mathbf{B}_{\mathrm{amb}}$, the cross product between the neutral barium beam velocity vector and the ambient magnetic field, and the $x$ axis is parallel to the cross product of the $y$ and $z$ axes, i.e., the $x$ axis is in the plane defined by $\mathbf{v}_{\text {beam }}$ and $\mathbf{B}_{\mathrm{amb}}$.

Figure 1 gives an overview of the main aspects of the momentum transfer in the CRIT II experiment. It also introduces some useful nomenclature, based on the observation that the injected ion beam acts like a generator in the ambient plasma rest frame, feeding energy into the ionosphere [6]. When the injected neutral atoms are ionized, they create a low-frequency generator region with a current density $\mathbf{i}_{G}$ perpendicular to the ambient 


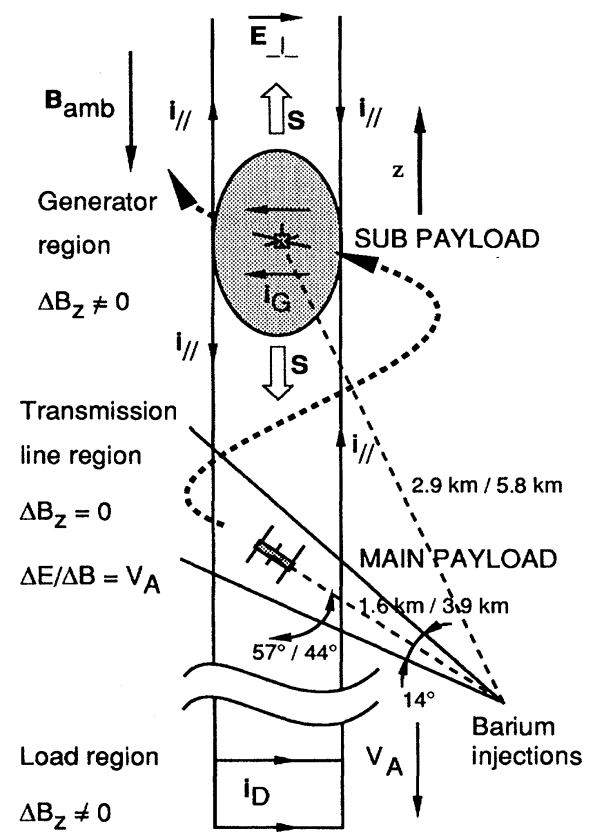

FIG. 1. The mass loading model for CRIT II. The neutral barium beams with an opening angle of $14^{\circ}$ are denoted by the solid lines originating at the point of explosion. The injected ions in the generator region are shown approximately one barium gyroperiod after ionization, when they have moved along the ambient magnetic field up to the subpayload (along the S-shaped dashed line). Also shown are the distances from the explosions to the payloads for burst 1 and burst 2 , respectively, as well as the angle between the barium beams and the ambient magnetic field.

magnetic field. $\mathbf{i}_{G}$ closes in the ambient plasma through magnetic-field-aligned currents $\mathbf{i}_{\|}$that are carried by shear Alfvén waves, and through displacement currents $\mathbf{i}_{D}$ in the fronts of these waves. The region between the generator region and the Alfvén wave fronts is often called the transmission line region, since no crossfield currents exist here $\left(\Delta B_{z}=0\right.$ when the $z$ axis is defined to be antiparallel to the ambient magnetic field). The normal relationship between the electric and magnetic field fluctuations in Alfvén waves, $\Delta E / \Delta B=$ $V_{A}$, should, however, hold. In the load region, the ambient plasma is being accelerated by a $\mathbf{i}_{D} \times \mathbf{B}_{\mathrm{amb}}$ force. The magnetic-field-aligned Poynting flux $\mathbf{S}$ is always directed away from the generator region. Due to the neutral beam's initial magnetic-field-aligned velocity component, the generator region will continue up along the magnetic field lines, and pass the subpayload as shown in Fig. 1.

The net generator current $\mathbf{i}_{G}$ consists of a number of different components, both parallel and perpendicular to the beam. There are low-frequency components that are caused directly by the gyromotion of the newly created barium ions, and there are also high-frequency components due to instability-driven processes. (We use the gyrofrequency of the ambient oxygen ions, $f_{g, \mathrm{O}^{+}}=$ $40 \mathrm{~Hz}$, to separate the two frequency regions.) Of course, only the low-frequency part of $\mathbf{i}_{G}$ is closed by means of Alfvén waves. The high-frequency components are closed through other mechanisms, possibly whistler waves. These components are, however, only marginally involved in the momentum transfer to the ambient plasma.

Figure 2 shows the subpayload magnetic field data from both bursts. The upper panel shows burst 1 , while the lower panel shows burst 2 . The vertical dashed lines refer to the times when the denser part of the fast neutral jet $(8-13.5 \mathrm{~km} / \mathrm{s})$ crosses the magnetic field line at which the subpayload is located.

Note in the upper panel of Fig. 2 the small $(\approx 5 \mathrm{nT})$ peak in $\Delta B_{z}$ at $t=0.12 \mathrm{~s}$. This peak coincides with the time when the neutral barium stream first reaches the field line of the subpayload (the left dashed line). Because field-aligned currents give magnetic perturbations only in the plane perpendicular to the ambient magnetic field $\mathbf{B}_{\mathrm{amb}}$, this small peak must be sign of a cross-field current. Since it occurs at a time when no barium ions have had time to reach the subpayload, we interpret it as a sign of the displacement current, $\mathbf{i}_{D}=\varepsilon_{p}\left(d \mathbf{E}_{\text {perp }} / d t\right)$, in the front of the Alfvén wave that is propagating away from the barium cloud. This interpretation is supported by a theoretical analysis based on Ampère's law, $\operatorname{curl} \mathbf{B}=$ $\mu_{0}$ i. Such an analysis using the CRIT II data results in

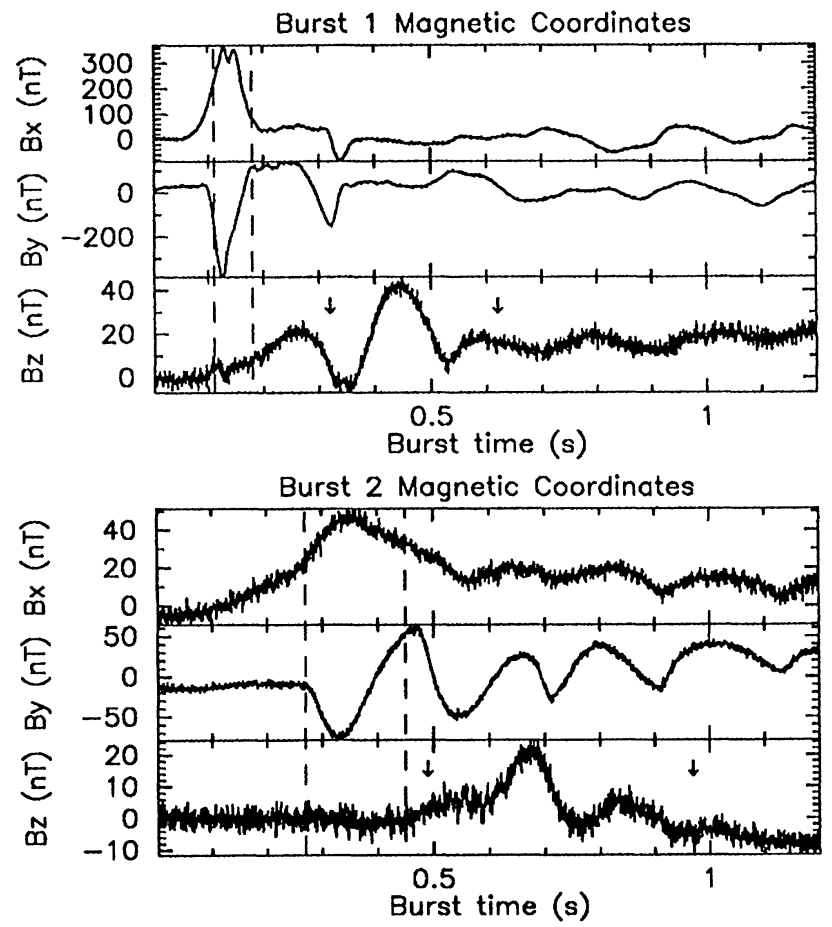

FIG. 2. The subpayload $\mathbf{B}$ field data in the magnetic coordinate system. 
an expected amplitude of $\Delta B_{z}$ due to the displacement current that is in the order of 5-10 nT, in good agreement with the observations.

At $t=0.32 \mathrm{~s}$ after burst 1 (Fig. 2, upper panel) an arrow in the $\Delta B_{z}$ panel indicates when the first barium ions reach the subpayload, while a second arrow at $t=$ $0.62 \mathrm{~s}$ indicates when the last ions from the fast part of the barium jet reach the subpayload, assuming that after ionization they continue up the field lines with the parallel velocity of the neutrals. Similar arrows have been drawn in the burst 2 subpayload data (Fig. 2, lower panel). The arrival of the barium ions is shown by a large $\Delta B_{z}$ component in the subpayload data. The $\Delta B_{z}$ components connected to the passage of the ion cloud do not coincide precisely with the arrows. The probable explanation is that the ions were scattered in velocity space during the ionization process, something which is confirmed by the particle detector measurements [10].

Based on these magnetic field data, we will now establish the geometry and strength of the momentum transfer current system, i.e., the low-frequency large scale current which flows to the distant ionosphere during the active phase (the phase when the fast part of the neutral barium stream passed the payload, and when the ionization of the barium ions took place). For this purpose the electric and magnetic field data from the main payload inside the stream is of little use. The main payload low-frequency electric field data were corrupted by a dc offset, which occurred during the active phase [5], and the interpretation of the main payload magnetic field data is complicated both by strong instability-associated currents and by the unknown geometry of the current system. At the subpayload the currents are constrained to flow along the magnetic field, and the analysis can be made. This is the first ionospheric CIV experiment in which such an analysis is possible. (The subpayload did not observe any of the strong high-frequency features seen in the main payload data. We therefore suggest that the high-frequency currents caused by the ionization process merged into larger structures in a matching region between the main and the subpayload.)

We assume that the generator current density $\mathbf{i}_{G}$ (see Fig. 1) is approximately homogeneous inside the generator region, and that this generator region coincides with the active region of the fast jet, defined as the region where strong wave activity and ionization were observed by the main payload [5]. $\mathbf{i}_{G}$ then results in field-aligned currents $\mathbf{i}_{\|}(x, y)$, which, in turn, give a magnetic field perturbation $\Delta \mathbf{B}_{G}$ above the generator region inside the flux tube through that region. $\Delta \mathbf{B}_{G}$ is turned $90^{\circ}$ with respect to $\mathbf{i}_{G}$.

Figure 3 shows $\mathbf{i}_{G}$ during the active phase of the two bursts, calculated using the subpayload $\Delta \mathbf{B}$ data. From a physical point of view it is suitable to separate the discussion of these currents into the beam-parallel component $\mathbf{i}_{G x}$ and the cross-beam component $\mathbf{i}_{G y}$. A beam-

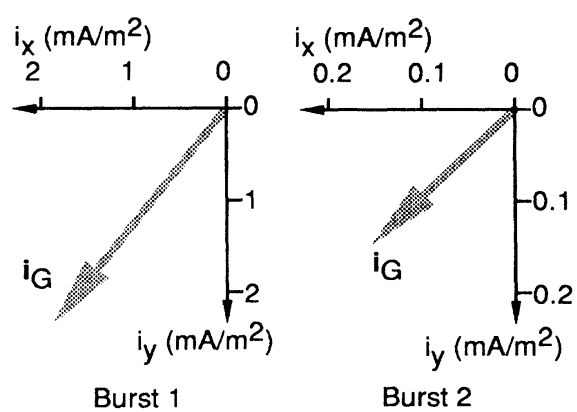

FIG. 3. The generator current in the two bursts, calculated from the subpayload magnetic field, and projected on the plane perpendicular to $\mathbf{B}_{\mathrm{amb}}$. The current is shown for $t=0.12 \mathrm{~s}$ in burst 1 , and $t=0.35 \mathrm{~s}$ in burst 2 .

parallel current component is expected already in the absence of CIV for an injected heavy ion cloud [2]. The current density can be directly estimated from the density increase measured inside the beam by the main payload as $i_{G x} \approx n_{\mathrm{Ba}}+v_{\mathrm{Ba} \text {,perp }} e$. Using estimates of $n_{\mathrm{Ba}^{+}}$ from [3], this gives $i_{G x}=0.6-1.3 \mathrm{~mA} / \mathrm{m}^{2}$ in burst 1 and $i_{G x}=0.13-0.22 \mathrm{~mA} / \mathrm{m}^{2}$ in burst 2 . For burst 2 , these $i_{G x}$ values agree well with the $i_{G x}$ components in Fig. 3 , while during burst 1 they are off by a factor of 2 . The $\mathbf{i}_{G x} \times \mathbf{B}_{\mathrm{amb}}$ force is simply the magnetic force on the ions which makes them gyrate; no energy exchange is involved and the connection to the CIV process is, therefore, only indirect.

The $\mathbf{i}_{G y} \times \mathbf{B}_{\mathrm{amb}}$ force, on the other hand, decelerates the injected ions and thus taps the beam energy. Already in the absence of CIV there should be such a component due to Hall drifting ambient electrons [2]. It should,

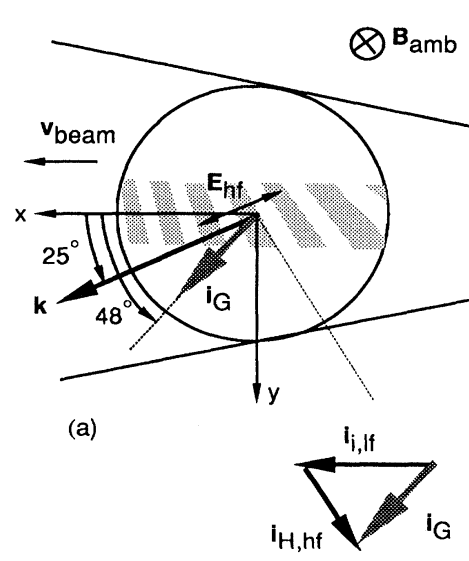

(b)

FIG. 4. Observations from CRIT II burst 1. (a) The $\mathbf{k}$ vector and the high-frequency electric field $\mathbf{E}_{\mathrm{hf}}$ in the generator region for CRIT II burst 1 , as well as the generator current $\mathbf{i}_{G}$ from Fig. 3. (b) A possible combination of ion and Hall currents that would add up to the measured generator current. 
however, be weak - an order of magnitude below the observed cross-beam currents.

Using results from the main payload [5], Fig. 4(a) shows the $\mathbf{k}$ vector and the high-frequency electric field $\mathbf{E}_{\mathrm{hf}}$ in the generator region for CRIT II burst 1, as well as the generator current $\mathbf{i}_{G}$ from Fig. 3. The angle between $\mathbf{k}$ and $\mathbf{i}_{G}$ shows that $\mathbf{i}_{G}$ has to be a combination of currents flowing along and across the instability wave fronts.

Figure 4(b) shows a possible combination of ion and Hall currents that would add up to the measured generator current. The currents $\mathbf{i}_{i, \text { lf }}$ (a low-frequency beam-parallel ion current) and $\mathbf{i}_{H, \text { hf }}$ (a high-frequency, instabilitydriven Hall current) drawn in Fig. 4(b) are not based on measurements, but are found by combining a beamparallel current with a Hall current flowing along the instability wave fronts, such that they add up to $\mathbf{i}_{G}$.

Based on Fig. 4, a model for the current systems and the momentum transfer in CRIT II emerges that has connections to both the homogeneous and the inhomogeneous model. In this new model, the macroscopic current along the injection direction is basically the low-frequency ion current described in [2]. The electric field set up by the ion motion forces the ambient electrons to Hall drift across the injection direction, and the relative motion between the injected ions and the drifting electrons results in an instability with a wave vector $\mathbf{k}$ directed along the direction of that relative motion. The high-frequency electric field caused by the instability drives strong Hall currents along the instability wave fronts, which then results in a net current at an angle to $\mathbf{k}$. This model gives a natural coupling between the energy and momentum transfer processes.

According to Fig. 4, the cross-beam current can be attributed mainly to the instability. The instability's effectiveness in tapping the kinetic energy of the ions can therefore be estimated as follows. The rate of change of the barium ion velocity is found from $d v_{\mathrm{Ba}^{+}} / d t=$ $\left|\mathbf{i}_{G y} \times \mathbf{B}_{\mathrm{amb}}\right| / m_{\mathrm{Ba}} n_{\mathrm{Ba}^{+}}$. This gives the time constant for ion energy loss $\tau_{\text {loss }}=W /(d W / d t) \approx 0.01 \mathrm{~s}$ in burst 1 , using parameters from [3]. This is definitely significant, since it is comparable to the time duration of the active region in burst 1 . Since the ions start with the beam velocity and therefore initially follow the active region, their residence time might even be longer than that. It seems likely that a substantial part of the ion energy is rapidly fed into the process, which maintains the transverse current $\mathbf{i}_{G y}$. This agrees very well with the ion data from the active region, which shows that the ions, starting as a beam with small velocity spread, very rapidly are scattered both in energy and in pitch angle [10].

The exact nature of the instability is, however, not yet entirely clear. From Fig. 4, we find it reasonable to assume that the Hall currents flow along the wave fronts, but that still remains to be proven. A new current component is also seen in the CRIT II data that has not been observed previously in any similar experiment. This is a high-frequency beam-parallel current that is caused by the instability through a modulation of the ion current.

It is obvious that more efforts are needed in order to fully understand the energy transfer mechanism. However, regarding momentum transfer and the connection between these two processes, it is our opinion that the results from CRIT II now give a rather complete picture.

This work was financed in part by the Swedish Natural Science Research Council.

[1] H. Alfvén, On the Origin of the Solar System (Oxford University Press, New York, 1954).

[2] N. Brenning, M. C. Kelley, J. Providakes, H. C. StenbaekNielsen, and C. Swenson, J. Geophys. Res. 96, 9735 (1991).

[3] N. Brenning, C. Swenson, M. C. Kelley, J. Providakes, and R. Torbert, Adv. Space Res. 12(12), 91 (1992).

[4] C. M. Swenson, M. C. Kelley, N. Brenning, R. Torbert, F. Primdahl, and K. D. Baker, Adv. Space Res. 12(12), 65 (1992).

[5] C. M. Swenson, thesis, Cornell University, Ithaca, 1992.

[6] G. Haerendel, Z. Naturforsch. A 37, 728 (1982).

[7] M. A. Raadu, Astrophys. Space Sci. 55, 125 (1978).

[8] A. Piel, E. Möbius, and G. Himmel, Astrophys. Space Sci. 72, 211 (1980).

[9] O. Bolin, Royal Institute of Technology, Stockholm, Report No. ALP-1994-107, 1994.

[10] R. Torbert (private communication). 